\title{
Strong convergence of three-step iteration methods for a countable family of generalized strict pseudocontractions in Hilbert spaces
}

\author{
Shi-Xiu Li, Lu-Chuan Ceng ${ }^{*}$, Hui-Ying Hu and Xiao-Jie Wang
}

"Correspondence:

zenglc@hotmail.com Department of Mathematics, Shanghai Normal University, Shanghai, 200234, China

\begin{abstract}
In this paper, we introduce a new class of generalized strict pseudocontractions in a real Hilbert space, and we consider a three-step Ishikawa-type iteration method

$$
\left\{\begin{array}{l}
z_{n}=\left(1-\gamma_{n}\right) x_{n}+\gamma_{n} T_{n} x_{n} \\
y_{n}=\left(1-\beta_{n}\right) x_{n}+\beta_{n} T_{n} z_{n} \\
x_{n+1}=\left(1-\alpha_{n}\right) x_{n}+\alpha_{n} T_{n} y_{n}
\end{array}\right.
$$

for finding a common fixed point of a countable family $\left\{T_{n}\right\}$ of uniformly Lipschitz generalized $\lambda_{n}$-strict pseudocontractions. Under mild conditions imposed on the parameter sequences $\left\{\alpha_{n}\right\},\left\{\beta_{n}\right\}$ and $\left\{\gamma_{n}\right\}$, we prove the strong convergence of $\left\{x_{n}\right\}$ to a common fixed point of a countable family $\left\{T_{n}\right\}$ of uniformly Lipschitz generalized strict pseudocontractions. On the other hand, we also introduce three-step hybrid viscosity approximation method for finding a common fixed point of a countable family $\left\{T_{n}\right\}$ of uniformly Lipschitz generalized $\lambda_{n}$-strict pseudocontractions with $\lambda_{n}=0$, i.e., a countable family $\left\{T_{n}\right\}$ of uniformly Lipschitz pseudocontractions. Under appropriate conditions we derive the strong convergence results for this method. The results presented in this paper improve and extend the corresponding results in the earlier and recent literature.
\end{abstract}

MSC: 47H06; 47H09; 47J20; 47J30

Keywords: generalized strict pseudocontraction; uniformly Lipschitz; uniformly closed; strong convergence; fixed point

\section{Introduction}

Let $C$ be a nonempty subset of $H$. A mapping $T: C \rightarrow H$ is said to be nonexpansive, if

$$
\|T x-T y\| \leq\|x-y\|, \quad \forall x, y \in C .
$$

A mapping $T: C \rightarrow H$ is called pseudocontractive if

$$
\langle T x-T y, x-y\rangle \leq\|x-y\|^{2}, \quad \forall x, y \in C .
$$

@2014 Li et al.; licensee Springer. This is an Open Access article distributed under the terms of the Creative Commons Attribution License (http://creativecommons.org/licenses/by/2.0), which permits unrestricted use, distribution, and reproduction in any medium, provided the original work is properly cited. 
Note that inequality (1.2) can be equivalently written as

$$
\|T x-T y\| \leq\|x-y\|^{2}+\|(I-T) x-(I-T) y\|^{2}, \quad \forall x, y \in C,
$$

where $I$ is the identity mapping on $H$.

A mapping $T: C \rightarrow H$ is called a strict pseudocontraction [1] if for all $x, y \in C$ there exists a constant $\lambda \in[0,1)$ such that

$$
\|T x-T y\|^{2} \leq\|x-y\|^{2}+\lambda\|(I-T) x-(I-T) y\|^{2}, \quad \forall x, y \in C .
$$

In this case, we also say that $T$ is a $\lambda$-strict pseudocontraction.

In this paper, we introduce and consider the concept of generalized strict pseudocontraction. A mapping $T: C \rightarrow H$ is called a generalized strict pseudocontraction if there exists a constant $\lambda \in[0,1)$ such that

$$
\|T x-T y\|^{2} \leq\|x-y\|^{2}+(1+\lambda)\|(I-T) x-(I-T) y\|^{2}, \quad \forall x, y \in C .
$$

In this case, we also say that $T$ is a generalized $\lambda$-strict pseudocontraction. It is remarkable that whenever $T: C \rightarrow H$ is a nonexpansive mapping, a pseudocontraction or a strict pseudocontraction, $T$ is certainly a generalized strict pseudocontraction.

Apart from their being an important generalization of nonexpansive mappings and strict pseudocontractions, interest in generalized strict pseudocontractions stems mainly from the fact that they are also an important generalization of pseudocontractions. It is well known that there exists a close connection between pseudocontractions and the important class of nonlinear monotone mappings, where a mapping $A$ with domain $D(A)$ and range $R(A)$ in $H$ is called monotone if

$$
\langle A x-A y, x-y\rangle \geq 0, \quad \forall x, y \in D(A) .
$$

We observe that $A$ is monotone if and only if $T:=I-A$ is pseudocontractive and thus a zero of $A, x \in N(A):=\{x \in D(A): A x=0\}$, is a fixed point of $T, x \in F(T):=\{x \in D(T): T x=x\}$. It is now well known (see, e.g. [2]) that if $A$ is monotone then the solutions of the equation $A x=0$ correspond to the equilibrium points of some evolution systems. Consequently, considerable research efforts, especially within the past 20 years or so, have been devoted to iterative methods for approximating fixed points of $T$ when $T$ is pseudocontractive (see, e.g., [3-5] and references therein).

The most general iterative algorithm for nonexpansive mappings studied by many authors is the following:

$$
x_{0} \in C, \quad x_{n+1}=\left(1-\alpha_{n}\right) x_{n}+\alpha_{n} T x_{n}, \quad n \geq 0,
$$

where $\left\{\alpha_{n}\right\} \subset(0,1)$ and satisfies the following additional assumptions: (i) $\lim _{n \rightarrow \infty} \alpha_{n}=0$; (ii) $\sum_{n=0}^{\infty} \alpha_{n}=\infty$, the sequence $\left\{x_{n}\right\}$ generated by (1.6) is generally referred to as the Mann iteration one in the light of Mann [6].

The Mann iteration process does not generally converge to a fixed point of $T$ even when the fixed point exists. If, for example, $C$ is a nonexpansive, and the Mann iteration process 
is defined by (1.6) with (i) $\lim _{n \rightarrow \infty} \alpha_{n}=0$; (ii) $\sum_{n=0}^{\infty} \alpha_{n}=\infty$, one can only prove that the sequence is an approximate fixed point sequence. That is, $\left\|x_{n}-T x_{n}\right\| \rightarrow 0$ as $n \rightarrow \infty$. To get the sequence $\left\{x_{n}\right\}$ to converge to a fixed point of $T$ (when such a fixed point exists), some type of compactness condition must be additionally imposed either on $C$ (e.g., $C$ is compact) or on $T$.

Later on, some authors tried to prove convergence of Mann iteration scheme to a fixed point of a much more general and important class of Lipschitz pseudocontractive mappings. But in 2001 Chidume and Mutangadura [7] gave an example of a Lipschitz pseudocontractive self-mapping on a compact convex subset of a Hilbert space with a unique fixed point for which no Mann sequence converges. Consequently, for this class of mappings, the Mann sequence may not converge to a fixed point of Lipschitz pseudocontractive mappings even when $C$ is a compact convex subset of $H$.

In 1974, Ishikawa [8] introduced an iteration process, which in some sense is more general than that of Mann and which converges to a fixed point of a Lipschitz pseudocontractive self-mapping $T$ on $C$. The following theorem is proved.

Theorem IS [8] If $C$ is a compact convex subset of a Hilbert space $H, T: C \rightarrow C$ is a Lipschitz pseudocontractive mapping and $x_{0}$ is any point of $C$, then the sequence $\left\{x_{n}\right\}$ converges strongly to a fixed point of $T$, where $\left\{x_{n}\right\}$ is defined iteratively for each integer $n \geq 0$ by

$$
\left\{\begin{array}{l}
y_{n}=\left(1-\beta_{n}\right) x_{n}+\beta_{n} T x_{n} \\
x_{n+1}=\left(1-\alpha_{n}\right) x_{n}+\alpha_{n} T y_{n}
\end{array}\right.
$$

where $\left\{\alpha_{n}\right\},\left\{\beta_{n}\right\}$ are sequences of positive numbers satisfying the conditions:

(i) $0 \leq \alpha_{n} \leq \beta_{n} \leq 1$; (ii) $\lim _{n \rightarrow \infty} \beta_{n}=0$; (iii) $\sum_{n=1}^{\infty} \alpha_{n} \beta_{n}=\infty$.

The iteration method of Theorem IS, which is now referred to as the Ishikawa iterative method has been studied extensively by various authors. But it is still an open question whether or not this method can be employed to approximate fixed points of Lipschitz pseudocontractive mappings without the compactness assumption on $C$ or $T$ (see, e.g., $[4,9,10])$.

In order to obtain a strong convergence theorem for pseudocontractive mappings without the compactness assumption, Zhou [11] established the hybrid Ishikawa algorithm for Lipschitz pseudocontractive mappings as follows:

$$
\left\{\begin{aligned}
y_{n}= & \left(1-\alpha_{n}\right) x_{n}+\alpha_{n} T x_{n}, \\
z_{n}= & \left(1-\beta_{n}\right) x_{n}+\beta_{n} T y_{n}, \\
C_{n}= & \left\{z \in C:\left\|z_{n}-z\right\|^{2} \leq\left\|x_{n}-z\right\|^{2}\right. \\
& \left.-\alpha_{n} \beta_{n}\left(1-2 \alpha_{n}-L^{2} \alpha_{n}^{2}\right)\left\|x_{n}-T x_{n}\right\|^{2}\right\}, \\
Q_{n}= & \left\{z \in C:\left\langle x_{n}-z, x_{0}-x_{n}\right\rangle \geq 0\right\}, \\
x_{n+1}= & P_{C_{n} \cap Q_{n}} x_{0}, \quad n \in N .
\end{aligned}\right.
$$

He proved that the sequence $\left\{x_{n}\right\}$ defined by (1.8) converges strongly to $P_{F(T)} x_{0}$, where $P_{C}$ is the metric projection from $H$ into $C$. We observe that the iterative algorithm (1.8) generates a sequence $\left\{x_{n}\right\}$ by projecting $x_{0}$ onto the intersection of closed convex sets $C_{n}$ and $Q_{n}$ for each $n \geq 0$. 
In 2009, Yao et al. [12] introduced the hybrid Mann algorithm as follows. Let $C$ be a nonempty, closed, and convex subset of a real Hilbert space $H$. Let $T: C \rightarrow C$ be a $L$-Lipschitz pseudocontractive mapping such that $F(T) \neq \emptyset$. Assume that the sequence $\left\{\alpha_{n}\right\} \subset[a, b]$ for some $a, b \in\left(0, \frac{1}{1+L}\right)$. Then for $C_{1}=C$ and $x_{1}=P_{C_{1}} x_{0}$, they proved that the sequence $\left\{x_{n}\right\}$ defined by

$$
\left\{\begin{array}{l}
y_{n}=\left(1-\alpha_{n}\right) x_{n}+\alpha_{n} T x_{n} \\
C_{n+1}=\left\{z \in C_{n}:\left\|\alpha_{n}(I-T) y_{n}\right\|^{2} \leq 2 \alpha_{n}\left\langle x_{n}-z,(I-T) y_{n}\right\rangle\right\} \\
x_{n+1}=P_{C_{n+1}} x_{0}, \quad n \in N
\end{array}\right.
$$

converges strongly to $P_{F(T)} x_{0}$.

More recently, Tang et al. [13] generalized algorithm (1.9) to the hybrid Ishikawa iterative process. Let $C$ be a nonempty, closed, and convex subset of a real Hilbert space $H$. Let $T: C \rightarrow C$ be a Lipschitz pseudocontractive mapping. Let $\left\{\alpha_{n}\right\}$ and $\left\{\beta_{n}\right\}$ be sequences in $[0,1]$. Suppose that $x_{0} \in H$. For $C_{1}=C$ and $x_{1}=P_{C_{1}} x_{0}$, define a sequence $\left\{x_{n}\right\}$ of $C$ as follows:

$$
\left\{\begin{aligned}
y_{n}= & \left(1-\alpha_{n}\right) x_{n}+\alpha_{n} T z_{n}, \\
z_{n}= & \left(1-\beta_{n}\right) x_{n}+\beta_{n} T x_{n}, \\
C_{n+1}= & \left\{z \in C_{n}:\left\|\alpha_{n}(I-T) y_{n}\right\|^{2} \leq 2 \alpha_{n}\left\langle x_{n}-z,(I-T) y_{n}\right\rangle\right. \\
& \left.+2 \alpha_{n} \beta_{n}\left\|x_{n}-T x_{n}\right\|\left\|y_{n}-x_{n}+\alpha_{n}(I-T) y_{n}\right\|\right\}, \\
x_{n+1}= & P_{C_{n+1}} x_{0}, \quad n \in N .
\end{aligned}\right.
$$

Then they proved that the hybrid algorithm (1.10) strongly converges to a fixed point of the Lipschitz pseudocontractive mapping $T$. It is worth mentioning that the schemes in (1.8)-(1.10) are not easy to compute. They involve the computation of the intersection of $C_{n}$ and $Q_{n}$ for each $n \geq 1$.

Recently, Zegeye et al. [14] generalized algorithm (1.10) to Ishikawa iterative process (not hybrid) as follows. Let $C$ be a nonempty, closed, and convex subset of a real Hilbert space $H$. Let $\left\{T_{i}\right\}_{1 \leq i \leq N}$ be a finite family of Lipschitz pseudocontractive mappings with Lipschitzian constants $L_{i}$ for $i=1,2, \ldots, N$. Assume that the interior of $F:=\bigcap_{i=1}^{N} F\left(T_{i}\right)$ is nonempty. Let $\left\{x_{n}\right\}$ be a sequence generated from an arbitrary $x_{0} \in C$ by

$$
\left\{\begin{array}{l}
y_{n}=\left(1-\beta_{n}\right) x_{n}+\beta_{n} T_{n} x_{n} \\
x_{n+1}=\left(1-\alpha_{n}\right) x_{n}+\alpha_{n} T_{n} y_{n}
\end{array}\right.
$$

Under appropriate conditions, they proved that $\left\{x_{n}\right\}$ converges strongly to $x^{*} \in F$.

Very recently, Cheng and $\mathrm{Su}$ [15] generalized algorithm (1.11) to three-step Ishikawatype iterative process. Let $C$ be a nonempty, closed, and convex subset of a real Hilbert space $H$, let $\left\{T_{n}\right\}_{n=1}^{\infty}: C \rightarrow C$ be a countable family of uniformly closed and uniformly Lipschitz pseudocontractive mappings with Lipschitz constants $L_{n}$. Let $L:=\sup _{n \geq 1} L_{n}<$ $\infty$. Assume that the interior of $F:=\bigcap_{n=1}^{\infty} F\left(T_{n}\right)$ is nonempty. Let $\left\{x_{n}\right\}$ be a sequence generated from an arbitrary $x_{1} \in C$ by the following algorithm:

$$
\left\{\begin{array}{l}
z_{n}=\left(1-\gamma_{n}\right) x_{n}+\gamma_{n} T_{n} x_{n} \\
y_{n}=\left(1-\beta_{n}\right) x_{n}+\beta_{n} T_{n} z_{n} \\
x_{n+1}=\left(1-\alpha_{n}\right) x_{n}+\alpha_{n} T_{n} y_{n} .
\end{array}\right.
$$

Under mild conditions, they proved that $\left\{x_{n}\right\}$ converges strongly to $x^{*} \in F$. 
Our concern now is the following: Is it possible to prove strong convergence of threestep Ishikawa-type iterative algorithm (1.12) for finding a common fixed point of a countable family $\left\{T_{n}\right\}$ of uniformly Lipschitz generalized strict pseudocontractive mappings?

In this paper, we consider and analyze three-step Ishikawa-type iterative algorithm (1.12) for finding a common fixed point of a countable family $\left\{T_{n}\right\}$ of uniformly Lipschitz generalized $\lambda_{n}$-strict pseudocontractions. Under mild conditions imposed on the parameter sequences $\left\{\alpha_{n}\right\},\left\{\beta_{n}\right\}$ and $\left\{\gamma_{n}\right\}$, we prove the strong convergence of $\left\{x_{n}\right\}$ to a common fixed point of a countable family $\left\{T_{n}\right\}$ of uniformly Lipschitz generalized strict pseudocontractions. On the other hand, inspired by the viscosity approximation method [16] we also introduce a three-step hybrid viscosity approximation method for finding a common fixed point of a countable family $\left\{T_{n}\right\}$ of uniformly Lipschitz generalized $\lambda_{n}$-strict pseudocontractions with $\lambda_{n}=0$, i.e., a countable family $\left\{T_{n}\right\}$ of uniformly Lipschitz pseudocontractions. Under appropriate conditions we derive the strong convergence results for this method. The results presented in this paper improve and extend the corresponding results in the earlier and recent literature; for instance, Zhou [17], Yao et al. [12], Tang et al. [13], Cheng and Su [15] and Zegeye et al. [14].

\section{Preliminaries}

Let $C$ be a nonempty subset of a real Hilbert space $H$. A mapping $T: C \rightarrow H$ is called Lipschitz continuous if there exists a constant $L>0$ such that

$$
\|T x-T y\| \leq L\|x-y\|, \quad \forall x, y \in C .
$$

If $L=1$, then $T$ is called nonexpansive; and if $L<1$, then $T$ is called a contraction. It is easy to see from (2.1) that every contraction mapping is nonexpansive and every nonexpansive mapping is Lipschitz.

A countable family of mappings $\left\{T_{n}\right\}_{n \geq 1}: C \rightarrow H$ is called uniformly Lipschitz with Lipschitz constants $L_{n}>0, n \geq 1$, if there exists $L=\sup _{n \geq 1} L_{n} \in(0, \infty)$ such that

$$
\left\|T_{n} x-T_{n} y\right\| \leq L\|x-y\|, \quad \forall x, y \in C, n \geq 1 .
$$

A countable family of mapping $\left\{T_{n}\right\}_{n \geq 1}: C \rightarrow H$ is called uniformly closed if $x_{n} \rightarrow x^{*}$ and $\left\|x_{n}-T_{n} x_{n}\right\| \rightarrow 0$ imply $x^{*} \in \bigcap_{n=1}^{\infty} F\left(T_{n}\right)$.

In the sequel, we also need the following definition and lemma.

Let $H$ be a real Hilbert space. Define the function $\phi: H \times H \rightarrow R$ as follows:

$$
\phi(x, y):=\|x-y\|^{2}=\|x\|^{2}-2\langle x, y\rangle+\|y\|^{2}, \quad \forall x, y \in H ;
$$

it was studied previously by Alber [18], Kamimula and Takahashi [19] and Reich [20].

It is clear from the definition of the function $\phi$ that

$$
(\|x\|-\|y\|)^{2} \leq \phi(x, y) \leq(\|x\|+\|y\|)^{2}, \quad \forall x, y \in H .
$$

The function $\phi$ also has the following property:

$$
\phi(y, x)=\phi(y, z)+\phi(z, x)+2\langle z-y, x-z\rangle, \quad \forall x, y, z \in H .
$$


Lemma 2.1 [21] Let $H$ be a real Hilbert space. Then

$$
\|\alpha x+(1-\alpha) y\|^{2}=\alpha\|x\|^{2}+(1-\alpha)\|y\|^{2}-\alpha(1-\alpha)\|x-y\|^{2}, \quad \forall x, y \in H, \alpha \in[0,1] .
$$

The following lemma is a direct consequence of the inner product. Thus, its proof is omitted.

Lemma 2.2 Let $H$ be a real Hilbert space. Then

$$
\|x\|^{2}+2\langle y, x\rangle \leq\|x+y\|^{2} \leq\|x\|^{2}+2\langle y, x+y\rangle, \quad \forall x, y \in H .
$$

Lemma 2.3 [22, p.303] Let $\left\{a_{n}\right\}$ and $\left\{b_{n}\right\}$ be two sequences of nonnegative real numbers satisfying the inequality

$$
a_{n+1} \leq a_{n}+b_{n}, \quad \forall n \geq 0 .
$$

If $\sum_{n=0}^{\infty} b_{n}<\infty$, then $\lim _{n \rightarrow \infty} a_{n}$ exists.

\section{Uniformly Lipschitz generalized strict pseudocontractions}

In this section, we consider and analyze three-step Ishikawa-type iteration method introduced by Cheng and Su [15] for finding a common fixed point of a countable family of uniformly closed and uniformly Lipschitz generalized $\lambda_{n}$-strict pseudocontractive mappings with Lipschitz constants $L_{n}$ in a real Hilbert space.

Theorem 3.1 Let C be a nonempty, closed, and convex subset of a real Hilbert space H. Let $\left\{T_{n}\right\}_{n \geq 1}: C \rightarrow C$ be a countable family of uniformly closed and uniformly Lipschitz generalized $\lambda_{n}$-strict pseudocontractive mappings with Lipschitz constants $L_{n}$. Let $L:=\sup _{n \geq 1} L_{n}$. Assume that the interior of $F:=\bigcap_{n=1}^{\infty} F\left(T_{n}\right)$ is nonempty. Let $\left\{x_{n}\right\}$ be a sequence generated from an arbitrary $x_{1} \in C$ by the following algorithm:

$$
\left\{\begin{array}{l}
z_{n}=\left(1-\gamma_{n}\right) x_{n}+\gamma_{n} T_{n} x_{n}, \\
y_{n}=\left(1-\beta_{n}\right) x_{n}+\beta_{n} T_{n} z_{n}, \\
x_{n+1}=\left(1-\alpha_{n}\right) x_{n}+\alpha_{n} T_{n} y_{n},
\end{array}\right.
$$

where $\left\{\alpha_{n}\right\},\left\{\beta_{n}\right\}$, and $\left\{\gamma_{n}\right\}$ are sequences in $(0,1)$ satisfying the following conditions:

(i) $\left\{\alpha_{n}\right\},\left\{\beta_{n}\right\},\left\{\gamma_{n}\right\} \subset[a, b]$ for some $a, b \in(0,1)$;

(ii) $\alpha_{n} \leq \beta_{n} \leq \gamma_{n}$ and $3 \beta_{n}-2 \beta_{n}^{2}-\gamma_{n} \leq 0$ for all $n \geq 1$;

(iii) $\sup _{n \geq 1} \gamma_{n} \leq \gamma$ with $\gamma^{3} L^{4}+2 \gamma^{2} L^{3}+\gamma^{2} L^{2}+2 \gamma L^{2}+2 \gamma<1$;

(iv) $\sum_{n=1}^{\infty} \lambda_{n}<\infty$.

Then $\left\{x_{n}\right\}$ converges strongly to $x^{*} \in F$ provided $\sup _{n \geq 1}\left\{\left\|x_{n}-T_{n} x_{n}\right\|+\left\|y_{n}-T_{n} y_{n}\right\|\right\}<\infty$.

Proof Let $p \in F$. Utilizing Lemma 2.1, we obtain from (1.5) and (3.1)

$$
\begin{aligned}
\left\|x_{n+1}-p\right\|^{2}= & \left\|\left(1-\alpha_{n}\right)\left(x_{n}-p\right)+\alpha_{n}\left(T_{n} y_{n}-p\right)\right\|^{2} \\
= & \left(1-\alpha_{n}\right)\left\|x_{n}-p\right\|^{2}+\alpha_{n}\left\|T_{n} y_{n}-p\right\|^{2}-\alpha_{n}\left(1-\alpha_{n}\right)\left\|x_{n}-T_{n} y_{n}\right\|^{2} \\
\leq & \left(1-\alpha_{n}\right)\left\|x_{n}-p\right\|^{2}+\alpha_{n}\left[\left\|y_{n}-p\right\|^{2}+\left(1+\lambda_{n}\right)\left\|y_{n}-T_{n} y_{n}\right\|^{2}\right] \\
& -\alpha_{n}\left(1-\alpha_{n}\right)\left\|x_{n}-T_{n} y_{n}\right\|^{2}
\end{aligned}
$$




$$
\begin{aligned}
= & \left(1-\alpha_{n}\right)\left\|x_{n}-p\right\|^{2}+\alpha_{n}\left\|y_{n}-p\right\|^{2}+\alpha_{n}\left\|y_{n}-T_{n} y_{n}\right\|^{2} \\
& -\alpha_{n}\left(1-\alpha_{n}\right)\left\|x_{n}-T_{n} y_{n}\right\|^{2}+\alpha_{n} \lambda_{n}\left\|y_{n}-T_{n} y_{n}\right\|^{2}, \\
\left\|y_{n}-p\right\|^{2}= & \left(1-\beta_{n}\right)\left\|x_{n}-p\right\|^{2}+\beta_{n}\left\|T_{n} z_{n}-p\right\|^{2}-\beta_{n}\left(1-\beta_{n}\right)\left\|x_{n}-T_{n} z_{n}\right\|^{2} \\
\leq & \left(1-\beta_{n}\right)\left\|x_{n}-p\right\|^{2}+\beta_{n}\left[\left\|z_{n}-p\right\|^{2}+\left(1+\lambda_{n}\right)\left\|z_{n}-T_{n} z_{n}\right\|^{2}\right] \\
& -\beta_{n}\left(1-\beta_{n}\right)\left\|x_{n}-T_{n} z_{n}\right\|^{2} \\
= & \left(1-\beta_{n}\right)\left\|x_{n}-p\right\|^{2}+\beta_{n}\left\|z_{n}-p\right\|^{2}+\beta_{n}\left\|z_{n}-T_{n} z_{n}\right\|^{2} \\
& -\beta_{n}\left(1-\beta_{n}\right)\left\|x_{n}-T_{n} z_{n}\right\|^{2}+\beta_{n} \lambda_{n}\left\|z_{n}-T_{n} z_{n}\right\|^{2}
\end{aligned}
$$

and

$$
\begin{aligned}
\left\|z_{n}-p\right\|^{2}= & \left(1-\gamma_{n}\right)\left\|x_{n}-p\right\|^{2}+\gamma_{n}\left\|T_{n} x_{n}-p\right\|^{2}-\gamma_{n}\left(1-\gamma_{n}\right)\left\|x_{n}-T_{n} x_{n}\right\|^{2} \\
\leq & \left(1-\gamma_{n}\right)\left\|x_{n}-p\right\|^{2}+\gamma_{n}\left[\left\|x_{n}-p\right\|^{2}+\left(1+\lambda_{n}\right)\left\|x_{n}-T_{n} x_{n}\right\|^{2}\right] \\
& -\gamma_{n}\left(1-\gamma_{n}\right)\left\|x_{n}-T_{n} x_{n}\right\|^{2} \\
= & \left\|x_{n}-p\right\|^{2}+\gamma_{n}\left\|x_{n}-T_{n} x_{n}\right\|^{2}-\gamma_{n}\left(1-\gamma_{n}\right)\left\|x_{n}-T_{n} x_{n}\right\|^{2}+\gamma_{n} \lambda_{n}\left\|x_{n}-T_{n} x_{n}\right\|^{2} \\
= & \left\|x_{n}-p\right\|^{2}+\gamma_{n}^{2}\left\|x_{n}-T_{n} x_{n}\right\|^{2}+\gamma_{n} \lambda_{n}\left\|x_{n}-T_{n} x_{n}\right\|^{2} .
\end{aligned}
$$

In addition, utilizing (3.1), we have

$$
\begin{aligned}
\left\|z_{n}-T_{n} z_{n}\right\|^{2} & =\left\|\left(1-\gamma_{n}\right)\left(x_{n}-T_{n} z_{n}\right)+\gamma_{n}\left(T_{n} x_{n}-T_{n} z_{n}\right)\right\|^{2} \\
& =\left(1-\gamma_{n}\right)\left\|x_{n}-T_{n} z_{n}\right\|^{2}+\gamma_{n}\left\|T_{n} x_{n}-T_{n} z_{n}\right\|^{2}-\gamma_{n}\left(1-\gamma_{n}\right)\left\|x_{n}-T_{n} x_{n}\right\|^{2} \\
& \leq\left(1-\gamma_{n}\right)\left\|x_{n}-T_{n} z_{n}\right\|^{2}+\gamma_{n} L^{2}\left\|x_{n}-z_{n}\right\|^{2}-\gamma_{n}\left(1-\gamma_{n}\right)\left\|x_{n}-T_{n} x_{n}\right\|^{2} \\
& =\left(1-\gamma_{n}\right)\left\|x_{n}-T_{n} z_{n}\right\|^{2}+\gamma_{n}^{3} L^{2}\left\|x_{n}-T_{n} x_{n}\right\|^{2}-\gamma_{n}\left(1-\gamma_{n}\right)\left\|x_{n}-T_{n} x_{n}\right\|^{2} \\
& =\left(1-\gamma_{n}\right)\left\|x_{n}-T_{n} z_{n}\right\|^{2}+\gamma_{n}\left(\gamma_{n}^{2} L^{2}+\gamma_{n}-1\right)\left\|x_{n}-T_{n} x_{n}\right\|^{2} .
\end{aligned}
$$

Substituting (3.4) and (3.5) into (3.3), we obtain

$$
\begin{aligned}
\left\|y_{n}-p\right\|^{2} \leq & \left(1-\beta_{n}\right)\left\|x_{n}-p\right\|^{2}+\beta_{n}\left(\left\|x_{n}-p\right\|^{2}+\gamma_{n}^{2}\left\|x_{n}-T_{n} x_{n}\right\|^{2}+\gamma_{n} \lambda_{n}\left\|x_{n}-T_{n} x_{n}\right\|^{2}\right) \\
& +\beta_{n}\left[\left(1-\gamma_{n}\right)\left\|x_{n}-T_{n} z_{n}\right\|^{2}+\gamma_{n}\left(\gamma_{n}^{2} L^{2}+\gamma_{n}-1\right)\left\|x_{n}-T_{n} x_{n}\right\|^{2}\right] \\
& -\beta_{n}\left(1-\beta_{n}\right)\left\|x_{n}-T_{n} z_{n}\right\|^{2}+\beta_{n} \lambda_{n}\left\|z_{n}-T_{n} z_{n}\right\|^{2} \\
= & \left\|x_{n}-p\right\|^{2}+\beta_{n} \gamma_{n}\left(\gamma_{n}^{2} L^{2}+2 \gamma_{n}-1\right)\left\|x_{n}-T_{n} x_{n}\right\|^{2} \\
& +\beta_{n}\left(\beta_{n}-\gamma_{n}\right)\left\|x_{n}-T_{n} z_{n}\right\|^{2}+\beta_{n} \lambda_{n}\left(1-\gamma_{n}\right)\left\|x_{n}-T_{n} z_{n}\right\|^{2} \\
& +\beta_{n} \lambda_{n} \gamma_{n}\left(\gamma_{n}^{2} L^{2}+\gamma_{n}\right)\left\|x_{n}-T_{n} x_{n}\right\|^{2} .
\end{aligned}
$$

We observe that

$$
\begin{aligned}
\left\|y_{n}-T_{n} y_{n}\right\|^{2} & =\left\|\left(1-\beta_{n}\right)\left(x_{n}-T_{n} y_{n}\right)+\beta_{n}\left(T_{n} z_{n}-T_{n} y_{n}\right)\right\|^{2} \\
& =\left(1-\beta_{n}\right)\left\|x_{n}-T_{n} y_{n}\right\|^{2}+\beta_{n}\left\|T_{n} z_{n}-T_{n} y_{n}\right\|^{2}-\beta_{n}\left(1-\beta_{n}\right)\left\|x_{n}-T_{n} z_{n}\right\|^{2} \\
& \leq\left(1-\beta_{n}\right)\left\|x_{n}-T_{n} y_{n}\right\|^{2}+\beta_{n} L^{2}\left\|z_{n}-y_{n}\right\|^{2}-\beta_{n}\left(1-\beta_{n}\right)\left\|x_{n}-T_{n} z_{n}\right\|^{2}
\end{aligned}
$$


and

$$
\begin{aligned}
\left\|z_{n}-y_{n}\right\| & =\left\|\left(1-\gamma_{n}\right) x_{n}+\gamma_{n} T_{n} x_{n}-\left(1-\beta_{n}\right) x_{n}-\beta_{n} T_{n} z_{n}\right\| \\
& =\left\|\beta_{n} x_{n}-\gamma_{n} x_{n}+\gamma_{n} T_{n} x_{n}-\beta_{n} T_{n} z_{n}\right\| \\
& =\left\|\left(\beta_{n}-\gamma_{n}\right) x_{n}-\left(\beta_{n}-\gamma_{n}\right) T_{n} x_{n}+\beta_{n}\left(T_{n} x_{n}-T_{n} z_{n}\right)\right\| \\
& \leq\left(\gamma_{n}-\beta_{n}\right)\left\|x_{n}-T_{n} x_{n}\right\|+\beta_{n} L\left\|x_{n}-z_{n}\right\| \\
& =\left(\gamma_{n}-\beta_{n}\right)\left\|x_{n}-T_{n} x_{n}\right\|+\beta_{n} \gamma_{n} L\left\|x_{n}-T_{n} x_{n}\right\| \\
& =\left(\gamma_{n}-\beta_{n}+\beta_{n} \gamma_{n} L\right)\left\|x_{n}-T_{n} x_{n}\right\| .
\end{aligned}
$$

Thus, substituting (3.8) into (3.7), we get

$$
\begin{aligned}
\left\|y_{n}-T_{n} y_{n}\right\|^{2} \leq & \left(1-\beta_{n}\right)\left\|x_{n}-T_{n} y_{n}\right\|^{2}-\beta_{n}\left(1-\beta_{n}\right)\left\|x_{n}-T_{n} z_{n}\right\|^{2} \\
& +\beta_{n} L^{2}\left(\gamma_{n}-\beta_{n}+\beta_{n} \gamma_{n} L\right)^{2}\left\|x_{n}-T_{n} x_{n}\right\|^{2} .
\end{aligned}
$$

Also, substituting (3.6) and (3.9) into (3.2), we have

$$
\begin{aligned}
\left\|x_{n+1}-p\right\|^{2} \leq & \left(1-\alpha_{n}\right)\left\|x_{n}-p\right\|^{2}+\alpha_{n}\left[\left\|x_{n}-p\right\|^{2}+\beta_{n}\left(\beta_{n}-\gamma_{n}\right)\left\|x_{n}-T_{n} z_{n}\right\|^{2}\right. \\
& +\beta_{n} \gamma_{n}\left(\gamma_{n}^{2} L^{2}+2 \gamma_{n}-1\right)\left\|x_{n}-T_{n} x_{n}\right\|^{2}+\beta_{n} \lambda_{n}\left(1-\gamma_{n}\right)\left\|x_{n}-T_{n} z_{n}\right\|^{2} \\
& \left.+\beta_{n} \lambda_{n} \gamma_{n}\left(\gamma_{n}^{2} L^{2}+\gamma_{n}\right)\left\|x_{n}-T_{n} x_{n}\right\|^{2}\right] \\
& +\alpha_{n}\left[\left(1-\beta_{n}\right)\left\|x_{n}-T_{n} y_{n}\right\|^{2}+\beta_{n} L^{2}\left(\gamma_{n}-\beta_{n}+\beta_{n} \gamma_{n} L\right)^{2}\left\|x_{n}-T_{n} x_{n}\right\|^{2}\right. \\
& \left.-\beta_{n}\left(1-\beta_{n}\right)\left\|x_{n}-T_{n} z_{n}\right\|^{2}\right]-\alpha_{n}\left(1-\alpha_{n}\right)\left\|x_{n}-T_{n} y_{n}\right\|^{2} \\
& +\alpha_{n} \lambda_{n}\left[\left(1-\beta_{n}\right)\left\|x_{n}-T_{n} y_{n}\right\|^{2}+\beta_{n} L^{2}\left(\gamma_{n}-\beta_{n}+\beta_{n} \gamma_{n} L\right)^{2}\left\|x_{n}-T_{n} x_{n}\right\|^{2}\right. \\
& \left.-\beta_{n}\left(1-\beta_{n}\right)\left\|x_{n}-T_{n} z_{n}\right\|^{2}\right] \\
= & \left\|x_{n}-p\right\|^{2}+\left[\alpha_{n} \beta_{n} \gamma_{n}\left(\gamma_{n}^{2} L^{2}+2 \gamma_{n}-1\right)\right. \\
& \left.+\alpha_{n} \beta_{n} L^{2}\left(\gamma_{n}-\beta_{n}+\beta_{n} \gamma_{n} L\right)^{2}\right]\left\|x_{n}-T_{n} x_{n}\right\|^{2} \\
& +\alpha_{n}\left(\alpha_{n}-\beta_{n}\right)\left\|x_{n}-T_{n} y_{n}\right\|^{2}+\alpha_{n} \beta_{n}\left(2 \beta_{n}-\gamma_{n}-1\right)\left\|x_{n}-T_{n} z_{n}\right\|^{2} \\
& +\lambda_{n}\left[\alpha_{n} \beta_{n} \gamma_{n}\left(\gamma_{n}^{2} L^{2}+\gamma_{n}\right)+\alpha_{n} \beta_{n} L^{2}\left(\gamma_{n}-\beta_{n}+\beta_{n} \gamma_{n} L\right)^{2}\right]\left\|x_{n}-T_{n} x_{n}\right\|^{2} \\
& +\alpha_{n} \lambda_{n}\left(1-\beta_{n}\right)\left\|x_{n}-T_{n} y_{n}\right\|^{2}+\lambda_{n} \alpha_{n} \beta_{n}\left(\beta_{n}-\gamma_{n}\right)\left\|x_{n}-T_{n} z_{n}\right\|^{2} .
\end{aligned}
$$

Note that

$$
\begin{aligned}
\left\|x_{n}-T_{n} y_{n}\right\|^{2} & \leq 2\left\|x_{n}-y_{n}\right\|^{2}+2\left\|y_{n}-T_{n} y_{n}\right\|^{2} \\
& =2 \beta_{n}^{2}\left\|x_{n}-T_{n} z_{n}\right\|^{2}+2\left\|y_{n}-T_{n} y_{n}\right\|^{2} .
\end{aligned}
$$

Substituting (3.11) into (3.10), we obtain

$$
\begin{aligned}
\left\|x_{n+1}-p\right\|^{2} \leq & \left\|x_{n}-p\right\|^{2}+\left[\alpha_{n} \beta_{n} \gamma_{n}\left(\gamma_{n}^{2} L^{2}+2 \gamma_{n}-1\right)\right. \\
& \left.+\alpha_{n} \beta_{n} L^{2}\left(\gamma_{n}-\beta_{n}+\beta_{n} \gamma_{n} L\right)^{2}\right]\left\|x_{n}-T_{n} x_{n}\right\|^{2}
\end{aligned}
$$




$$
\begin{aligned}
& +\alpha_{n}\left(\alpha_{n}-\beta_{n}\right)\left\|x_{n}-T_{n} y_{n}\right\|^{2}+\alpha_{n} \beta_{n}\left(2 \beta_{n}-\gamma_{n}-1\right)\left\|x_{n}-T_{n} z_{n}\right\|^{2} \\
& +\lambda_{n}\left[\alpha_{n} \beta_{n} \gamma_{n}\left(\gamma_{n}^{2} L^{2}+\gamma_{n}\right)+\alpha_{n} \beta_{n} L^{2}\left(\gamma_{n}-\beta_{n}+\beta_{n} \gamma_{n} L\right)^{2}\right]\left\|x_{n}-T_{n} x_{n}\right\|^{2} \\
& +\alpha_{n} \lambda_{n}\left(1-\beta_{n}\right)\left[2 \beta_{n}^{2}\left\|x_{n}-T_{n} z_{n}\right\|^{2}+2\left\|y_{n}-T_{n} y_{n}\right\|^{2}\right] \\
& +\lambda_{n} \alpha_{n} \beta_{n}\left(\beta_{n}-\gamma_{n}\right)\left\|x_{n}-T_{n} z_{n}\right\|^{2} \\
= & \left\|x_{n}-p\right\|^{2}+\left[\alpha_{n} \beta_{n} \gamma_{n}\left(\gamma_{n}^{2} L^{2}+2 \gamma_{n}-1\right)\right. \\
& \left.+\alpha_{n} \beta_{n} L^{2}\left(\gamma_{n}-\beta_{n}+\beta_{n} \gamma_{n} L\right)^{2}\right]\left\|x_{n}-T_{n} x_{n}\right\|^{2} \\
& +\alpha_{n}\left(\alpha_{n}-\beta_{n}\right)\left\|x_{n}-T_{n} y_{n}\right\|^{2}+\alpha_{n} \beta_{n}\left(2 \beta_{n}-\gamma_{n}-1\right)\left\|x_{n}-T_{n} z_{n}\right\|^{2} \\
& +\lambda_{n}\left[\alpha_{n} \beta_{n} \gamma_{n}\left(\gamma_{n}^{2} L^{2}+\gamma_{n}\right)+\alpha_{n} \beta_{n} L^{2}\left(\gamma_{n}-\beta_{n}+\beta_{n} \gamma_{n} L\right)^{2}\right]\left\|x_{n}-T_{n} x_{n}\right\|^{2} \\
& +\lambda_{n}\left[2\left(1-\beta_{n}\right) \alpha_{n} \beta_{n}^{2}+\alpha_{n} \beta_{n}\left(1-\gamma_{n}\right)-\alpha_{n} \beta_{n}\left(1-\beta_{n}\right)\right]\left\|x_{n}-T_{n} z_{n}\right\|^{2} \\
& +2 \lambda_{n}\left(1-\beta_{n}\right)\left\|y_{n}-T_{n} y_{n}\right\|^{2} .
\end{aligned}
$$

In terms of condition (iii) we have

$$
\gamma\left(\gamma^{2} L^{2}+\gamma L^{2}+2 \gamma-1\right)+\gamma^{2} L^{2}(1+\gamma L)^{2}<0
$$

which implies that

$$
\begin{aligned}
& \gamma_{n}\left(\gamma_{n}^{2} L^{2}+2 \gamma_{n}-1\right)+L^{2}\left(\gamma_{n}-\beta_{n}+\beta_{n} \gamma_{n} L\right)^{2} \\
& \quad \leq \gamma_{n}\left(\gamma_{n}^{2} L^{2}+\gamma_{n} L^{2}+2 \gamma_{n}-1\right)+L^{2}\left(\gamma_{n}+\gamma_{n}^{2} L\right)^{2}<0 .
\end{aligned}
$$

So,

$$
\alpha_{n} \beta_{n} \gamma_{n}\left(\gamma_{n}^{2} L^{2}+2 \gamma_{n}-1\right)+\alpha_{n} \beta_{n} L^{2}\left(\gamma_{n}-\beta_{n}+\beta_{n} \gamma_{n} L\right)^{2}<0 .
$$

Again from condition (ii), we have $\alpha_{n}-\beta_{n} \leq 0,2 \beta_{n}-\gamma_{n}-1 \leq 0$ and

$$
3 \beta_{n}-2 \beta_{n}^{2}-\gamma_{n} \leq 0
$$

Hence, we get

$$
\begin{aligned}
2 & \left(1-\beta_{n}\right) \alpha_{n} \beta_{n}^{2}+\alpha_{n} \beta_{n}\left(1-\gamma_{n}\right)-\alpha_{n} \beta_{n}\left(1-\beta_{n}\right) \\
& =2 \alpha_{n} \beta_{n}^{2}-2 \alpha_{n} \beta_{n}^{3}+\alpha_{n} \beta_{n}-\alpha_{n} \beta_{n} \gamma_{n}-\alpha_{n} \beta_{n}+\alpha_{n} \beta_{n}^{2} \\
& =3 \alpha_{n} \beta_{n}^{2}-2 \alpha_{n} \beta_{n}^{3}-\alpha_{n} \beta_{n} \gamma_{n} \\
& =\alpha_{n} \beta_{n}\left(3 \beta_{n}-2 \beta_{n}^{2}-\gamma_{n}\right) \leq 0,
\end{aligned}
$$

which, together with (3.12), implies that

$$
\begin{aligned}
\left\|x_{n+1}-p\right\|^{2} \leq & \left\|x_{n}-p\right\|^{2}+\left[\alpha_{n} \beta_{n} \gamma_{n}\left(\gamma_{n}^{2} L^{2}+2 \gamma_{n}-1\right)\right. \\
& \left.+\alpha_{n} \beta_{n} L^{2}\left(\gamma_{n}-\beta_{n}+\beta_{n} \gamma_{n} L\right)^{2}\right]\left\|x_{n}-T_{n} x_{n}\right\|^{2} \\
& +\lambda_{n}\left[\alpha_{n} \beta_{n} \gamma_{n}\left(\gamma_{n}^{2} L^{2}+\gamma_{n}\right)+\alpha_{n} \beta_{n} L^{2}\left(\gamma_{n}-\beta_{n}+\beta_{n} \gamma_{n} L\right)^{2}\right]\left\|x_{n}-T_{n} x_{n}\right\|^{2}
\end{aligned}
$$




$$
\begin{aligned}
& +2 \lambda_{n}\left(1-\beta_{n}\right)\left\|y_{n}-T_{n} y_{n}\right\|^{2} \\
\leq & \left\|x_{n}-p\right\|^{2}+\left[\alpha_{n} \beta_{n} \gamma_{n}\left(\gamma_{n}^{2} L^{2}+2 \gamma_{n}-1\right)\right. \\
& \left.+\alpha_{n} \beta_{n} L^{2}\left(\gamma_{n}-\beta_{n}+\beta_{n} \gamma_{n} L\right)^{2}\right]\left\|x_{n}-T_{n} x_{n}\right\|^{2} \\
& +\lambda_{n}\left[\left(L^{2}+1\right)+L^{2}(1+L)^{2}\right]\left\|x_{n}-T_{n} x_{n}\right\|^{2}+2 \lambda_{n}\left\|y_{n}-T_{n} y_{n}\right\|^{2} \\
\leq & \left\|x_{n}-p\right\|^{2}+\left[\alpha_{n} \beta_{n} \gamma_{n}\left(\gamma_{n}^{2} L^{2}+2 \gamma_{n}-1\right)\right. \\
& \left.+\alpha_{n} \beta_{n} L^{2}\left(\gamma_{n}-\beta_{n}+\beta_{n} \gamma_{n} L\right)^{2}\right]\left\|x_{n}-T_{n} x_{n}\right\|^{2}+\lambda_{n} M \\
\leq & \left\|x_{n}-p\right\|^{2}+\lambda_{n} M,
\end{aligned}
$$

where $\sup _{n \geq 1}\left\{\left(\left(L^{2}+1\right)+L^{2}(1+L)^{2}\right)\left\|x_{n}-T_{n} x_{n}\right\|^{2}+2\left\|y_{n}-T_{n} y_{n}\right\|^{2}\right\} \leq M$ for some $M>0$ (due to $\left.\sup _{n \geq 1}\left\{\left\|x_{n}-T_{n} x_{n}\right\|^{2}+\left\|y_{n}-T_{n} y_{n}\right\|^{2}\right\}<\infty\right)$. Consequently, we have

$$
\left\|x_{n+1}-p\right\|^{2} \leq\left\|x_{n}-p\right\|^{2}+\lambda_{n} M
$$

Utilizing condition (iv) and Lemma 2.3 we know that $\lim _{n \rightarrow \infty}\left\|x_{n}-p\right\|$ exists and hence $\left\{\left\|x_{n}-p\right\|\right\}$ is bounded. This implies that $\left\{x_{n}\right\},\left\{T_{n} x_{n}\right\},\left\{z_{n}\right\},\left\{T_{n} z_{n}\right\},\left\{y_{n}\right\}$, and $\left\{T_{n} y_{n}\right\}$ are also bounded.

On the other hand, from (2.3) we have

$$
\phi\left(p, x_{n}\right)=\phi\left(p, x_{n+1}\right)+\phi\left(x_{n+1}, x_{n}\right)+2\left\langle x_{n+1}-p, x_{n}-x_{n+1}\right\rangle,
$$

which implies that

$$
\left\langle x_{n+1}-p, x_{n}-x_{n+1}\right\rangle+\frac{1}{2} \phi\left(x_{n+1}, x_{n}\right)=\frac{1}{2}\left(\phi\left(p, x_{n}\right)-\phi\left(p, x_{n+1}\right)\right) .
$$

Since the interior of $F$ is nonempty, there exists $p^{*} \in F$ and $r>0$. Such that $p^{*}+r h \in F$ where $\|h\| \leq 1$. Thus, from the fact that $\phi(x, y)=\|x-y\|^{2}$, and from (3.14) and (3.15) we get

$$
\begin{aligned}
0 & \leq\left\langle x_{n+1}-\left(p^{*}+\gamma h\right), x_{n}-x_{n+1}\right\rangle+\frac{1}{2} \phi\left(x_{n+1}, x_{n}\right)+\frac{1}{2} \lambda_{n} M \\
& =\frac{1}{2}\left(\phi\left(p^{*}+\gamma h, x_{n}\right)-\phi\left(p^{*}+\gamma h, x_{n+1}\right)\right)+\frac{1}{2} \lambda_{n} M .
\end{aligned}
$$

Then from (3.15) and (3.16), we obtain

$$
\begin{aligned}
r\left\langle h, x_{n}-x_{n+1}\right\rangle & \leq\left\langle x_{n+1}-p^{*}, x_{n}-x_{n+1}\right\rangle+\frac{1}{2} \phi\left(x_{n+1}, x_{n}\right)+\frac{1}{2} \lambda_{n} M \\
& =\frac{1}{2}\left(\phi\left(p^{*}, x_{n}\right)-\phi\left(p^{*}, x_{n+1}\right)\right)+\frac{1}{2} \lambda_{n} M
\end{aligned}
$$

and hence

$$
\left\langle h, x_{n}-x_{n+1}\right\rangle \leq \frac{1}{2 r}\left(\phi\left(p^{*}, x_{n}\right)-\phi\left(p^{*}, x_{n+1}\right)\right)+\frac{1}{2 r} \lambda_{n} M .
$$

Since $h$ with $\|h\| \leq 1$ is arbitrary, we have

$$
\left\|x_{n}-x_{n+1}\right\| \leq \frac{1}{2 r}\left(\phi\left(p^{*}, x_{n}\right)-\phi\left(p^{*}, x_{n+1}\right)\right)+\frac{1}{2 r} \lambda_{n} M .
$$


So, whenever $n>m$, we get

$$
\begin{aligned}
\left\|x_{m}-x_{n}\right\| & =\left\|x_{m}-x_{m+1}+x_{m+1}-\cdots-x_{n-1}+x_{n-1}-x_{n}\right\| \\
& \leq \sum_{i=m}^{n-1}\left\|x_{i}-x_{i+1}\right\| \\
& \leq \frac{1}{2 r} \sum_{i=m}^{n-1}\left(\phi\left(p^{*}, x_{i}\right)-\phi\left(p^{*}, x_{i+1}\right)\right)+\frac{M}{2 r} \sum_{i=m}^{n-1} \lambda_{i} \\
& =\frac{1}{2 r}\left(\phi\left(p^{*}, x_{m}\right)-\phi\left(p^{*}, x_{n}\right)\right)+\frac{M}{2 r} \sum_{i=m}^{n-1} \lambda_{i} .
\end{aligned}
$$

Note that $\left\{\phi\left(p^{*}, x_{n}\right)\right\}$ converges and $\sum_{n=1}^{\infty} \lambda_{n}$ also converges. Thus, we find that $\left\{x_{n}\right\}$ is a Cauchy sequence. Since $C$ is a closed subset of $H$, there exists $x^{*} \in C$ such that

$$
\lim _{n \rightarrow \infty}\left\|x_{n}-x^{*}\right\|=0
$$

Furthermore, from (3.13) and conditions (i)-(iii), we get

$$
\begin{aligned}
a^{3} & {\left[\left(1-\gamma^{2} L^{2}-2 \gamma\right)-\gamma L^{2}(1+\gamma L)^{2}\right]\left\|x_{n}-T_{n} x_{n}\right\|^{2} } \\
& \leq \alpha_{n} \beta_{n} \gamma_{n}\left[\left(1-\gamma_{n}^{2} L^{2}-2 \gamma_{n}\right)-\gamma_{n} L^{2}\left(1+\gamma_{n} L\right)^{2}\right]\left\|x_{n}-T_{n} x_{n}\right\|^{2} \\
& =\left[\alpha_{n} \beta_{n} \gamma_{n}\left(1-\gamma_{n}^{2} L^{2}-2 \gamma_{n}\right)-\alpha_{n} \beta_{n} \gamma_{n}^{2} L^{2}\left(1+\gamma_{n} L\right)^{2}\right]\left\|x_{n}-T_{n} x_{n}\right\|^{2} \\
& \leq\left[\alpha_{n} \beta_{n} \gamma_{n}\left(1-\gamma_{n}^{2} L^{2}-2 \gamma_{n}\right)-\alpha_{n} \beta_{n} L^{2}\left(\gamma_{n}-\beta_{n}+\beta_{n} \gamma_{n} L\right)^{2}\right]\left\|x_{n}-T_{n} x_{n}\right\|^{2} \\
& \leq\left\|x_{n}-p\right\|^{2}-\left\|x_{n+1}-p\right\|^{2}+\lambda_{n} M .
\end{aligned}
$$

From $\lim _{n \rightarrow \infty} \lambda_{n}=0$ and the existence of $\lim _{n \rightarrow \infty}\left\|x_{n}-p\right\|$, it follows that

$$
\lim _{n \rightarrow \infty}\left\|x_{n}-T_{n} x_{n}\right\|=0
$$

Since $\left\{T_{n}\right\}_{n \geq 1}$ are uniformly closed, from (3.17) and (3.18), we deduce that $x^{*} \in F:=$ $\bigcap_{n=1}^{\infty} F\left(T_{n}\right)$. The proof is complete.

Remark 3.1 As previously, it is worth emphasizing that whenever $T: C \rightarrow H$ is a nonexpansive mapping, a pseudocontraction or a strict pseudocontraction, $T$ is certainly a generalized strict pseudocontraction. Here we provide an example to illustrate a countable family of uniformly closed and uniformly Lipschitz generalized strict pseudocontractions with the interior of the common fixed points being nonempty. Suppose that $X:=R$ and $C:=\left[-\frac{1}{2}, \frac{1}{2}\right] \subset R$. Let $\left\{T_{n}\right\}_{n \geq 1}: C \rightarrow C$ be defined by

$$
T_{n} x:= \begin{cases}x, & x \in\left[-\frac{1}{2}, 0\right), \\ \left(\frac{1}{3^{n}}+\frac{1}{3}\right) x, & x \in\left[0, \frac{1}{2}\right] .\end{cases}
$$

Then we observe that $F:=\bigcap_{n=1}^{\infty} F\left(T_{n}\right)=\left[-\frac{1}{2}, 0\right]$, and hence the interior of common fixed point set $F$ is nonempty. 
Next we show that $\left\{T_{n}\right\}_{n \geq 1}$ is a countable family of nonexpansive mappings. Indeed, suppose that $C_{1}=\left[-\frac{1}{2}, 0\right)$ and $C_{2}=\left[0, \frac{1}{2}\right]$.

If $x, y \in C_{1}$, we have

$$
\left\|T_{n} x-T_{n} y\right\|=\|x-y\| .
$$

If $x, y \in C_{2}$, we have

$$
\left\|T_{n} x-T_{n} y\right\|=\left(\frac{1}{3^{n}}+\frac{1}{3}\right)\|x-y\| \leq \frac{2}{3}\|x-y\| \leq\|x-y\| .
$$

If $x \in C_{1}, y \in C_{2}$, we have

$$
\begin{aligned}
\left\|T_{n} x-T_{n} y\right\| & =\left\|x-\left(\frac{1}{3^{n}}+\frac{1}{3}\right) y\right\| \\
& =-x+\left(\frac{1}{3^{n}}+\frac{1}{3}\right) y \\
& \leq-x+\frac{2}{3} y \\
& \leq-x+y \\
& =\|x-y\| .
\end{aligned}
$$

So, it follows that $\left\{T_{n}\right\}_{n \geq 1}$ is a sequence of nonexpansive mappings and hence uniformly Lipschitz with uniformly Lipschitz constant $L=\sup _{n \geq 1} L_{n}=1$.

Finally, we show that $\left\{T_{n}\right\}_{n \geq 1}$ is uniformly closed.

Case 1: If there exists $\left\{x_{n}\right\} \subset C_{1}$ such that $x_{n} \rightarrow x^{*} \in\left[-\frac{1}{2}, 0\right]$, and $\left\|x_{n}-T_{n} x_{n}\right\|=0$, we observe that $\left[-\frac{1}{2}, 0\right]=F$.

Case 2: If there exists $\left\{x_{n}\right\} \subset C_{2}$ such that $x_{n} \rightarrow x^{*} \in\left[0, \frac{1}{2}\right]$, then if and only if $x^{*}=0$ we have $\left\|x_{n}-T_{n} x_{n}\right\| \rightarrow 0$, and it is obvious that $0 \in F$.

If there exists $\left\{x_{n}\right\} \subset C$ :

(i) $\exists N$, as $n>N, x_{n} \in C_{2}$. The proof is the same as the proof of the second case.

(ii) $\exists N$, as $n>N, x_{n} \subset C_{1}$. The proof is the same as the proof of the first case.

(iii) $\left\{x_{n_{k}}\right\} \subset C_{1},\left\{x_{n_{j}}\right\} \subset C_{2}$. If there exists $x_{n} \rightarrow x^{*}$, then we have $x^{*}=0$. The proof is the same as the proof of the second case. So, we find that $\left\{T_{n}\right\}_{n \geq 1}$ is uniformly closed.

Remark 3.2 In Theorem 3.1, put $\alpha_{n} \equiv \frac{1}{28}, \beta_{n} \equiv \frac{1}{27}, \gamma_{n} \equiv \frac{1}{8}$ and take $L \in[1,1.5]$. Then conditions (i)-(iii) in Theorem 3.1 are satisfied. Indeed, it is clear that conditions (i)-(ii) in Theorem 3.1 are satisfied. Next we verify condition (iii) in Theorem 3.1. Observe that

$$
\begin{aligned}
\gamma^{3} L^{4}+2 \gamma^{2} L^{3}+\gamma^{2} L^{2}+2 \gamma L^{2}+2 \gamma \\
\quad \leq \frac{1.5^{4}}{8^{3}}+\frac{2 \times 1.5^{3}}{8^{2}}+\frac{1.5^{2}}{8^{2}}+\frac{2 \times 1.5^{2}+2}{8} \\
<\frac{2.3^{2}}{8^{3}}+\frac{3 \times 2.3}{8^{2}}+\frac{2.3}{8^{2}}+\frac{6.5}{8} \\
<\frac{5.3}{8^{3}}+\frac{9.2}{8^{2}}+\frac{6.5}{8}
\end{aligned}
$$




$$
\begin{aligned}
& <\frac{5.3}{512}+\frac{73.6}{512}+\frac{416}{512} \\
& =\frac{494.9}{512}<1 .
\end{aligned}
$$

Theorem 3.2 Let C be a nonempty, closed, and convex subset of a real Hilbert space H. Let $T_{n}: C \rightarrow C$ be a finite family of uniformly closed and uniformly Lipschitz generalized $\lambda_{n^{-}}$ strict pseudocontractive mappings with Lipschitzian constants $L_{n}, n=1,2, \ldots, N$. Let $L=$ $\sup _{n \geq 1} L_{n}$. Assume that the interior of $F:=\bigcap_{n=1}^{N} F\left(T_{n}\right)$ is nonempty. Let $\left\{x_{n}\right\}$ be a sequence generated from an arbitrary $x_{1} \in C$ by the following algorithm:

$$
\left\{\begin{array}{l}
z_{n}=\left(1-\gamma_{n}\right) x_{n}+\gamma_{n} T_{n} x_{n}, \\
y_{n}=\left(1-\beta_{n}\right) x_{n}+\beta_{n} T_{n} z_{n}, \\
x_{n+1}=\left(1-\alpha_{n}\right) x_{n}+\alpha_{n} T_{n} y_{n},
\end{array}\right.
$$

where $T_{n}:=T_{n(\bmod N)}$ and $\left\{\alpha_{n}\right\},\left\{\beta_{n}\right\}$, and $\left\{\gamma_{n}\right\}$ are sequences in $(0,1)$ satisfying the following conditions:

(i) $\left\{\alpha_{n}\right\},\left\{\beta_{n}\right\},\left\{\gamma_{n}\right\} \subset[a, b]$ for some $a, b \in(0,1)$;

(ii) $\alpha_{n} \leq \beta_{n} \leq \gamma_{n}$ and $3 \beta_{n}-2 \beta_{n}^{2}-\gamma_{n} \leq 0$ for all $n \geq 1$;

(iii) $\sup _{n \geq 1} \gamma_{n} \leq \gamma$ with $\gamma^{3} L^{4}+2 \gamma^{2} L^{3}+\gamma^{2} L^{2}+2 \gamma L^{2}+2 \gamma<1$.

Then $\left\{x_{n}\right\}$ converges strongly to $x^{*} \in F$ provided $\sup _{n \geq 1}\left\{\left\|x_{n}-T_{n} x_{n}\right\|+\left\|y_{n}-T_{n} y_{n}\right\|\right\}<\infty$.

If in Theorem 3.1, we consider a single Lipschitz generalized strict pseudocontractive mapping, then we get the following result.

Theorem 3.3 Let $C$ be a nonempty, closed, and convex subset of a real Hilbert space H. Let $T: C \rightarrow C$ be a Lipschitz generalized $\lambda$-strict pseudocontractive mapping with Lipschitzian constant L. Assume that the interior of $F(T)$ is nonempty. Let $\left\{x_{n}\right\}$ be a sequence generated from an arbitrary $x_{1} \in C$ by the following algorithm:

$$
\left\{\begin{array}{l}
z_{n}=\left(1-\gamma_{n}\right) x_{n}+\gamma_{n} T x_{n} \\
y_{n}=\left(1-\beta_{n}\right) x_{n}+\beta_{n} T z_{n} \\
x_{n+1}=\left(1-\alpha_{n}\right) x_{n}+\alpha_{n} T y_{n}
\end{array}\right.
$$

where $\left\{\alpha_{n}\right\},\left\{\beta_{n}\right\}$, and $\left\{\gamma_{n}\right\}$ are sequences in $(0,1)$ satisfying the following conditions:

(i) $\left\{\alpha_{n}\right\},\left\{\beta_{n}\right\},\left\{\gamma_{n}\right\} \subset[a, b]$ for some $a, b \in(0,1)$;

(ii) $\alpha_{n} \leq \beta_{n} \leq \gamma_{n}$ and $3 \beta_{n}-2 \beta_{n}^{2}-\gamma_{n} \leq 0$ for all $n \geq 1$;

(iii) $\sup _{n \geq 1} \gamma_{n} \leq \gamma$ with $\gamma^{3} L^{4}+2 \gamma^{2} L^{3}+\gamma^{2} L^{2}+2 \gamma L^{2}+2 \gamma<1$.

Then $\left\{x_{n}\right\}$ converges strongly to $x^{*} \in F(T)$ provided $\sup _{n \geq 1}\left\{\left\|x_{n}-T x_{n}\right\|+\left\|y_{n}-T y_{n}\right\|\right\}<\infty$.

Proof Following the same process as in the proof of Theorem 3.1, we obtain $x_{n} \rightarrow x^{*} \in$ $F(T)$.

Indeed, from (3.8) and conditions (i)-(iii), we deduce that

$$
\begin{aligned}
\alpha^{3} & {\left[\left(1-\gamma^{2} L^{2}-2 \gamma\right)-\gamma L^{2}(1+\gamma L)^{2}\right]\left\|x_{n}-T x_{n}\right\|^{2} } \\
& \leq \alpha_{n} \beta_{n} \gamma_{n}\left[\left(1-\gamma_{n}^{2} L^{2}-2 \gamma_{n}\right)-\gamma_{n} L^{2}\left(1+\gamma_{n} L\right)^{2}\right]\left\|x_{n}-T x_{n}\right\|^{2} \\
& =\left[\alpha_{n} \beta_{n} \gamma_{n}\left(1-\gamma_{n}^{2} L^{2}-2 \gamma_{n}\right)-\alpha_{n} \beta_{n} \gamma_{n}^{2} L^{2}\left(1+\gamma_{n} L\right)^{2}\right]\left\|x_{n}-T x_{n}\right\|^{2}
\end{aligned}
$$




$$
\begin{aligned}
& \leq\left[\alpha_{n} \beta_{n} \gamma_{n}\left(1-\gamma_{n}^{2} L^{2}-2 \gamma_{n}\right)-\alpha_{n} \beta_{n} L^{2}\left(\gamma_{n}-\beta_{n}+\beta_{n} \gamma_{n} L\right)^{2}\right]\left\|x_{n}-T x_{n}\right\|^{2} \\
& \leq\left\|x_{n}-p\right\|^{2}-\left\|x_{n+1}-p\right\|^{2}
\end{aligned}
$$

which yields

$$
\lim _{n \rightarrow \infty}\left\|x_{n}-T x_{n}\right\|=0
$$

and hence there exists a subsequence $\left\{x_{n_{k}}\right\}$ of $\left\{x_{n}\right\}$ such that

$$
\lim _{k \rightarrow \infty}\left\|x_{n_{k}}-T x_{n_{k}}\right\|=0
$$

Thus, $x_{n_{k}} \rightarrow x^{*}$ and the continuity of $T$ implies that $T x^{*}=x^{*} \in F(T)$.

\section{Uniformly Lipschitz pseudocontractions}

In this section, we introduce and analyze a three-step hybrid viscosity approximation method for finding a common fixed point of a countable family of uniformly closed and uniformly Lipschitz generalized $\lambda_{n}$-strict pseudocontractive mappings with $\lambda_{n} \equiv 0$, i.e., a countable family of uniformly closed and uniformly Lipschitz pseudocontractive mappings.

Theorem 4.1 Let $C$ be a nonempty, closed, and convex subset of a real Hilbert space $H$. Let $\left\{T_{n}\right\}_{n \geq 1}: C \rightarrow C$ be a countable family of uniformly closed and uniformly Lipschitz pseudocontractive mappings with Lipschitz constants $L_{n}$. Let $L=\sup _{n \geq 1} L_{n}$. Assume that the interior of $F:=\bigcap_{n=1}^{\infty} F\left(T_{n}\right)$ is nonempty. Let $\left\{x_{n}\right\}$ be a sequence generated from an arbitrary $x_{1} \in C$ by the following algorithm:

$$
\left\{\begin{array}{l}
z_{n}=\left(1-\gamma_{n}\right) x_{n}+\gamma_{n} T_{n} x_{n}, \\
y_{n}=\left(1-\beta_{n}\right) x_{n}+\beta_{n} T_{n} z_{n}, \\
x_{n+1}=\left(1-\alpha_{n}\right) y_{n}+\alpha_{n} f\left(x_{n}\right),
\end{array}\right.
$$

where $f: C \rightarrow C$ is a contractive mapping with contractive constant $\delta \in(0,1)$ and $\left\{\alpha_{n}\right\}$, $\left\{\beta_{n}\right\}$, and $\left\{\gamma_{n}\right\}$ are sequences in $(0,1)$ satisfying the following conditions:

(i) $\sum_{n=1}^{\infty} \alpha_{n}<\infty$;

(ii) $0<\beta \leq \beta_{n} \leq \gamma_{n}$;

(iii) $0<\gamma_{n} \leq \frac{-1+\sqrt{1+(1-\varepsilon) L^{2}}}{L^{2}}$ for some $\varepsilon \in(0,1)$.

Then $\left\{x_{n}\right\}$ converges strongly to $x^{*} \in F$.

Proof Let $p \in F$. Then from (4.1) and Lemma 2.2, we have

$$
\begin{aligned}
\left\|x_{n+1}-p\right\|^{2} & =\left\|\left(1-\alpha_{n}\right)\left(y_{n}-p\right)+\alpha_{n}\left(f\left(x_{n}\right)-f(p)\right)+\alpha_{n}(f(p)-p)\right\|^{2} \\
& \leq\left\|\left(1-\alpha_{n}\right)\left(y_{n}-p\right)+\alpha_{n}\left(f\left(x_{n}\right)-f(p)\right)\right\|^{2}+2 \alpha_{n}\left\langle f(p)-p, x_{n+1}-p\right\rangle \\
& \leq\left(1-\alpha_{n}\right)\left\|y_{n}-p\right\|^{2}+\alpha_{n}\left\|f\left(x_{n}\right)-f(p)\right\|^{2}+2 \alpha_{n}\left\langle f(p)-p, x_{n+1}-p\right\rangle \\
& \leq\left(1-\alpha_{n}\right)\left\|y_{n}-p\right\|^{2}+\alpha_{n} \delta\left\|x_{n}-p\right\|^{2}+\alpha_{n}\left(\|f(p)-p\|^{2}+\left\|x_{n+1}-p\right\|^{2}\right), \\
\left\|x_{n+1}-p\right\|^{2} & \leq\left\|y_{n}-p\right\|^{2}+\frac{\alpha_{n} \delta}{1-\alpha_{n}}\left\|x_{n}-p\right\|^{2}+\frac{\alpha_{n}}{1-\alpha_{n}}\|f(p)-p\|^{2},
\end{aligned}
$$




$$
\begin{aligned}
\left\|y_{n}-p\right\|^{2}= & \left(1-\beta_{n}\right)\left\|x_{n}-p\right\|^{2}+\beta_{n}\left\|T_{n} z_{n}-p\right\|^{2}-\beta_{n}\left(1-\beta_{n}\right)\left\|x_{n}-T_{n} z_{n}\right\|^{2} \\
\leq & \left(1-\beta_{n}\right)\left\|x_{n}-p\right\|^{2}+\beta_{n}\left\|z_{n}-p\right\|^{2}+\beta_{n}\left\|z_{n}-T_{n} z_{n}\right\|^{2} \\
& -\beta_{n}\left(1-\beta_{n}\right)\left\|x_{n}-T_{n} z_{n}\right\|^{2} \\
\leq & \left(1-\beta_{n}\right)\left\|x_{n}-p\right\|^{2}+\beta_{n}\left\|z_{n}-p\right\|^{2}+\beta_{n}\left\|z_{n}-T_{n} z_{n}\right\|^{2}, \\
\left\|z_{n}-p\right\|^{2}= & \left(1-\gamma_{n}\right)\left\|x_{n}-p\right\|^{2}+\gamma_{n}\left\|T_{n} x_{n}-p\right\|^{2}-\gamma_{n}\left(1-\gamma_{n}\right)\left\|x_{n}-T_{n} x_{n}\right\|^{2} \\
\leq & \left(1-\gamma_{n}\right)\left\|x_{n}-p\right\|^{2}+\gamma_{n}\left\|x_{n}-p\right\|^{2}+\gamma_{n}\left\|x_{n}-T_{n} x_{n}\right\|^{2} \\
= & -\gamma_{n}\left(1-\gamma_{n}\right)\left\|x_{n}-T_{n} x_{n}\right\|^{2} \\
= & \left\|x_{n}-p\right\|^{2}+\gamma_{n}^{2}\left\|x_{n}-T_{n} x_{n}\right\|^{2}-\gamma_{n}\left(1-\gamma_{n}\right)\left\|x_{n}-T_{n} x_{n}\right\|^{2}
\end{aligned}
$$

and

$$
\begin{aligned}
\left\|z_{n}-T_{n} z_{n}\right\|^{2} & =\left\|\left(1-\gamma_{n}\right)\left(x_{n}-T_{n} z_{n}\right)+\gamma_{n}\left(T_{n} x_{n}-T_{n} z_{n}\right)\right\|^{2} \\
& =\left(1-\gamma_{n}\right)\left\|x_{n}-T_{n} z_{n}\right\|^{2}+\gamma_{n}\left\|T_{n} x_{n}-T_{n} z_{n}\right\|^{2}-\gamma_{n}\left(1-\gamma_{n}\right)\left\|x_{n}-T_{n} x_{n}\right\|^{2} \\
& \leq\left(1-\gamma_{n}\right)\left\|x_{n}-T_{n} z_{n}\right\|^{2}+\gamma_{n} L^{2}\left\|x_{n}-z_{n}\right\|^{2}-\gamma_{n}\left(1-\gamma_{n}\right)\left\|x_{n}-T_{n} x_{n}\right\|^{2} \\
& =\left(1-\gamma_{n}\right)\left\|x_{n}-T_{n} z_{n}\right\|^{2}+\gamma_{n}^{3} L^{2}\left\|x_{n}-T_{n} x_{n}\right\|^{2}-\gamma_{n}\left(1-\gamma_{n}\right)\left\|x_{n}-T_{n} x_{n}\right\|^{2} \\
& =\left(1-\gamma_{n}\right)\left\|x_{n}-T_{n} z_{n}\right\|^{2}+\gamma_{n}\left(\gamma_{n}^{2} L^{2}+\gamma_{n}-1\right)\left\|x_{n}-T_{n} x_{n}\right\|^{2} .
\end{aligned}
$$

Substituting (4.4) and (4.5) into (4.3) we have

$$
\begin{aligned}
\left\|y_{n}-p\right\|^{2} \leq & \left(1-\beta_{n}\right)\left\|x_{n}-p\right\|^{2}+\beta_{n}\left(\left\|x_{n}-p\right\|^{2}+\gamma_{n}^{2}\left\|x_{n}-T_{n} x_{n}\right\|^{2}\right) \\
& +\beta_{n}\left[\left(1-\gamma_{n}\right)\left\|x_{n}-T_{n} z_{n}\right\|^{2}+\gamma_{n}\left(\gamma_{n}^{2} L^{2}+\gamma_{n}-1\right)\left\|x_{n}-T_{n} x_{n}\right\|^{2}\right] \\
& -\beta_{n}\left(1-\beta_{n}\right)\left\|x_{n}-T_{n} z_{n}\right\|^{2} \\
= & \left\|x_{n}-p\right\|^{2}+\beta_{n} \gamma_{n}\left(\gamma_{n}^{2} L^{2}+2 \gamma_{n}-1\right)\left\|x_{n}-T_{n} x_{n}\right\|^{2} \\
& +\beta_{n}\left(\beta_{n}-\gamma_{n}\right)\left\|x_{n}-T_{n} z_{n}\right\|^{2} \\
\leq & \left\|x_{n}-p\right\|^{2}+\beta_{n} \gamma_{n}\left(\gamma_{n}^{2} L^{2}+2 \gamma_{n}-1\right)\left\|x_{n}-T_{n} x_{n}\right\|^{2} .
\end{aligned}
$$

Since $0<\beta \leq \beta_{n} \leq \gamma_{n}$ and $\gamma_{n}^{2} L^{2}+2 \gamma_{n}-1 \leq-\varepsilon$ (due to condition (iii)), for some $\varepsilon \in(0,1)$, from (4.6) we get

$$
\left\|y_{n}-p\right\|^{2} \leq\left\|x_{n}-p\right\|^{2}-\beta_{n}^{2} \varepsilon\left\|x_{n}-T_{n} x_{n}\right\|^{2},
$$

which leads to

$$
\left\|y_{n}-p\right\|^{2} \leq\left\|x_{n}-p\right\|^{2}
$$

Thus,

$$
\begin{aligned}
\left\|x_{n+1}-p\right\| & \leq\left(1-\alpha_{n}\right)\left\|y_{n}-p\right\|+\alpha_{n}\left(\delta\left\|x_{n}-p\right\|+\|f(p)-p\|\right) \\
& \leq\left(1-\alpha_{n}(1-\delta)\right)\left\|x_{n}-p\right\|+\alpha_{n}\|f(p)-p\| \\
& \leq \max \left\{\left\|x_{0}-p\right\|, \frac{\|f(p)-p\|}{1-\delta}\right\} .
\end{aligned}
$$


Thus, $\left\{\left\|x_{n}-p\right\|\right\}$ is bounded. This implies that $\left\{x_{n}\right\},\left\{T_{n} x_{n}\right\},\left\{z_{n}\right\},\left\{T_{n} z_{n}\right\},\left\{y_{n}\right\},\left\{T_{n} y_{n}\right\}$, and $\left\{f\left(x_{n}\right)\right\}$ are also bounded.

Note that

$$
\begin{aligned}
\left\|x_{n+1}-p\right\|^{2} & =\left\|\left(1-\alpha_{n}\right)\left(y_{n}-p\right)+\alpha_{n}\left(f\left(x_{n}\right)-p\right)\right\|^{2} \\
& \leq\left(1-\alpha_{n}\right)\left\|y_{n}-p\right\|^{2}+\alpha_{n}\left\|f\left(x_{n}\right)-p\right\|^{2} \\
& \leq\left\|y_{n}-p\right\|^{2}+\alpha_{n}\left\|f\left(x_{n}\right)-p\right\|^{2} \\
& \leq\left\|x_{n}-p\right\|^{2}-\beta_{n}^{2} \varepsilon\left\|x_{n}-T_{n} x_{n}\right\|^{2}+\alpha_{n}\left\|f\left(x_{n}\right)-p\right\|^{2} .
\end{aligned}
$$

Hence it immediately follows that

$$
\left\|x_{n+1}-p\right\|^{2} \leq\left\|x_{n}-p\right\|^{2}+\alpha_{n}\left\|f\left(x_{n}\right)-p\right\|^{2}
$$

By Lemma 2.3, we conclude from $\sum_{n=1}^{\infty} \alpha_{n}<\infty$ and the boundedness of $\left\{x_{n}\right\},\left\{f\left(x_{n}\right)\right\}$ that $\lim _{n \rightarrow \infty}\left\|x_{n}-p\right\|$ exists.

Following the same process as in the proof of Theorem 3.1, we can derive

$$
x_{n} \rightarrow x^{*} \in C \text {. }
$$

Furthermore, from (4.8) and conditions (i), (ii), and (iii), we get

$$
\beta_{n}^{2} \varepsilon\left\|x_{n}-T_{n} x_{n}\right\|^{2} \leq\left\|x_{n}-p\right\|^{2}-\left\|x_{n+1}-p\right\|^{2}+\alpha_{n}\left\|f\left(x_{n}\right)-p\right\|^{2},
$$

which, together with $\lim _{n \rightarrow \infty} \alpha_{n}=0$, implies that

$$
\lim _{n \rightarrow \infty}\left\|x_{n}-T_{n} x_{n}\right\|=0
$$

Since the $\left\{T_{n}\right\}_{n=1}^{\infty}$ are uniformly closed, from (4.9) and (4.10) we infer that $x^{*} \in F:=$ $\bigcap_{n=1}^{\infty} F\left(T_{n}\right)=F$. The proof is complete.

Theorem 4.2 Let $H$ be a real Hilbert space and let $\left\{A_{n}\right\}_{n \geq 1}: H \rightarrow H$ be a countable family of uniformly Lipschitz monotone mappings with Lipschitzian constants $L_{n}$. Let $L:=\sup _{n \geq 1} L_{n}$. Assume that if $\left\|A_{n} x_{n}\right\| \rightarrow 0$ and $x_{n} \rightarrow x$, then $x \in \bigcap_{n=1}^{\infty} N\left(A_{n}\right)$. Let the interior of $F:=\bigcap_{n=1}^{\infty} N\left(A_{n}\right)$ be nonempty. Let $\left\{x_{n}\right\}$ be a sequence generated from an arbitrary $x_{1} \in H$ by the following algorithm:

$$
\left\{\begin{array}{l}
z_{n}=x_{n}-\gamma_{n} A_{n} x_{n}, \\
y_{n}=x_{n}-\beta_{n}\left(x_{n}-z_{n}\right)-\beta_{n} A_{n} z_{n}, \\
x_{n+1}=\left(1-\alpha_{n}\right) y_{n}+\alpha_{n} f\left(x_{n}\right),
\end{array}\right.
$$

where $f: H \rightarrow H$ is a contractive mapping with contractive constant $\delta \in(0,1)$, and $\left\{\alpha_{n}\right\}$, $\left\{\beta_{n}\right\}$, and $\left\{\gamma_{n}\right\}$ are sequences in $(0,1)$ satisfying the following conditions:

(i) $\sum_{n=1}^{\infty} \alpha_{n}<\infty$;

(ii) $0<\beta \leq \beta_{n} \leq \gamma_{n}$;

(iii) $0<\gamma_{n} \leq \frac{-1+\sqrt{1+(1-\varepsilon) L^{2}}}{L^{2}}$ for some $\varepsilon \in(0,1)$.

Then $\left\{x_{n}\right\}$ converges strongly to $x^{*} \in F$. 
Proof Put $T_{n}:=I-A_{n}$ for $n \geq 1$. Then we know that $\left\{T_{n}\right\}_{n \geq 1}$ is a countable family of uniformly closed and uniformly Lipschitz pseudocontractive mappings with $\bigcap_{n=1}^{\infty} F\left(T_{n}\right)=$ $\bigcap_{n=1}^{\infty} N\left(A_{n}\right) \neq \emptyset$. In this case, the iterative scheme (4.1) reduces to scheme (3.1) and hence the conclusion follows from Theorem 4.1.

Corollary 4.1 Let $H$ be a real Hilbert space and let $A_{n}: H \rightarrow H$ be a finite family of uniformly Lipschitz monotone mappings with Lipschitzian constants $L_{n}, n=1,2, \ldots, N$. Assume that if $\left\|A_{n} x_{n}\right\| \rightarrow 0$ and $x_{n} \rightarrow x$, then $x \in \bigcap_{n=1}^{N} N\left(A_{n}\right)$. Let the interior of $F:=$ $\bigcap_{n=1}^{N} N\left(A_{n}\right)$ be nonempty. Let $\left\{x_{n}\right\}$ be a sequence generated from an arbitrary $x_{1} \in H$ by the following algorithm:

$$
\left\{\begin{array}{l}
z_{n}=x_{n}-\gamma_{n} A_{n} x_{n} \\
y_{n}=x_{n}-\beta_{n}\left(x_{n}-z_{n}\right)-\beta_{n} A_{n} z_{n} \\
x_{n+1}=\left(1-\alpha_{n}\right) y_{n}+\alpha_{n} f\left(x_{n}\right)
\end{array}\right.
$$

where $A_{n}:=A_{n(\bmod N)}, f: H \rightarrow H$ is a contractive mapping with contractive constant $\delta \in$ $(0,1)$, and $\left\{\alpha_{n}\right\},\left\{\beta_{n}\right\}$, and $\left\{\gamma_{n}\right\}$ are sequences in $(0,1)$ satisfying the following conditions:

(i) $\sum_{n=1}^{\infty} \alpha_{n}<\infty$;

(ii) $0<\beta \leq \beta_{n} \leq \gamma_{n}$;

(iii) $0<\gamma_{n} \leq \frac{-1+\sqrt{1+(1-\varepsilon) L^{2}}}{L^{2}}$ for some $\varepsilon \in(0,1)$ and $L:=\max \left\{L_{n}: n=1,2, \ldots, N\right\}$.

Then $\left\{x_{n}\right\}$ converges strongly to $x^{*} \in F$.

Corollary 4.2 Let $H$ be a real Hilbert space, let $A: H \rightarrow H$ be a Lipschitz monotone mapping with Lipschitzian constant L. Assume that the interior of $N(A)$ is nonempty. Let $\left\{x_{n}\right\}$ be a sequence generated from an arbitrary $x_{1} \in H$ by the following algorithm:

$$
\left\{\begin{array}{l}
z_{n}=x_{n}-\gamma_{n} A x_{n} \\
y_{n}=x_{n}-\beta_{n}\left(x_{n}-z_{n}\right)-\beta_{n} A z_{n} \\
x_{n+1}=\left(1-\alpha_{n}\right) y_{n}+\alpha_{n} f\left(x_{n}\right)
\end{array}\right.
$$

where $f: H \rightarrow H$ is a contractive mapping with contractive constant $\delta \in(0,1)$, and $\left\{\alpha_{n}\right\}$, $\left\{\beta_{n}\right\}$, and $\left\{\gamma_{n}\right\}$ are sequences in $(0,1)$ satisfying the following conditions:

(i) $\sum_{n=1}^{\infty} \alpha_{n}<\infty$;

(ii) $0<\beta \leq \beta_{n} \leq \gamma_{n}$;

(iii) $0<\gamma_{n} \leq \frac{-1+\sqrt{1+(1-\varepsilon) L^{2}}}{L^{2}}$ for some $\varepsilon \in(0,1)$.

Then $\left\{x_{n}\right\}$ converges strongly to $x^{*} \in N(A)$.

The authors declare that they have no competing interests.

\section{Authors' contributions}

All authors contributed equally and significantly in writing this article. All authors read and approved the final manuscript.

\section{Acknowledgements}

All the authors were supported by the National Science Foundation of China (11071169) and PhD Program Foundation of Ministry of Education of China (20123127110002). 


\section{References}

1. Browder, FE, Petryshyn, WV: Construction of fixed points of nonlinear mappings in Hilbert spaces. J. Math. Anal. Appl. 20, 197-228 (1967)

2. Zeidler, E: Nonlinear Functional Analysis and Its Applications, Part II: Monotone Operators. Springer, Berlin (1985)

3. Chidume, CE, Moore, C: The solution by iteration of nonlinear equations in uniformly smooth Banach spaces. J. Math. Anal. Appl. 215, 132-146 (1997)

4. Liu, Q: The convergence theorems of the sequence of Ishikawa iterates for hemi-contractive mappings. J. Math. Anal. Appl. 148, 55-62 (1990)

5. Zhang, S: On the convergence problems of Ishikawa and Mann iteration processes with error for $\phi$-pseudocontractive type mappings. Appl. Math. Mech. 21, 1-10 (2000)

6. Mann, WR: Mean value methods in iteration. Proc. Am. Math. Soc. 4, 506-510 (1953)

7. Chidume, CE, Mutangadura, SA: An example of the Mann iteration method for Lipschitz pseudocontractions. Proc. Am. Math. Soc. 129, 2359-2363 (2001)

8. Ishikawa, S: Fixed points by a new iteration method. Proc. Am. Math. Soc. 44, 147-150 (1974)

9. Chidume, CE, Moore, C: Fixed point iteration for pseudocontractive maps. Proc. Am. Math. Soc. 127, $1163-1170$ (1999)

10. Liu, Q: On Naimpally and Singh's open question. J. Math. Anal. Appl. 124, 157-164 (1987)

11. Zhou, $\mathrm{H}$ : Convergence theorems of fixed points for Lipschitz pseudo-contractions in Hilbert spaces. J. Math. Anal. Appl. 343, 546-556 (2008)

12. Yao, YH, Liou, YC, Marino, G: A hybrid algorithm for pseudo-contractive mappings. Nonlinear Anal. 71, 4997-5002 (2009)

13. Tang, YC, Peng, JG, Liu, LW: Strong convergence theorem for pseudo-contractive mappings in Hilbert spaces. Nonlinear Anal. 74, 380-385 (2011)

14. Zegeye, H, Shahzad, N, Alghamdi, MA: Convergence of Ishikawa's iteration method for pseudocontractive mappings. Nonlinear Anal. 74, 7304-7311 (2011)

15. Cheng, QQ, Su, YF: Convergence theorems of a three-step iteration method for pseudocontrative mappings. Fixed Point Theory Appl. 2013, 100 (2013). doi:10.1186/1687-1812-2013-100

16. Xu, HK: Viscosity approximation methods for nonexpansive mappings. J. Math. Anal. Appl. 298, 279-291 (2004)

17. Zhou, $\mathrm{H}$ : Convergence theorems for $\lambda$-strict pseudocontractions in 2-uniformly smooth Banach spaces. Nonlinear Anal. 69, 3160-3173 (2008)

18. Alber, Y: Metric and generalized projection operators in Banach spaces: properties and applications. In: Kartsatos, AG (ed.) Theory and Applications of Nonlinear Operators of Accretive and Monotone Type. Lecture Notes in Pure and Appl. Math., vol. 178, pp. 15-50. Dekker, New York (1996)

19. Kamimura, S, Takahashi, W: Strong convergence of proximal-type algorithm in a Banach space. SIAM J. Optim. 13, 938-945 (2002)

20. Reich, S: A weak convergence theorem for the alternating method with Bergman distance. In: Kartsatos, AG (ed.) Theory and Applications of Nonlinear Operators of Accretive and Monotone Type. Lecture Notes in Pure and Appl. Math., vol. 178, pp. 313-318. Dekker, New York (1996)

21. Takahashi, W: Introduction to Nonlinear and Convex Analysis. Yokohama Publishers, Yokohama (2009)

22. Tan, KK, Xu, HK: Approximating fixed points of nonexpansive mapping by the Ishikawa iteration process. J. Math. Anal. Appl. 178, 301-308 (1993)

10.1186/1687-1812-2014-66

Cite this article as: Li et al.: Strong convergence of three-step iteration methods for a countable family of

generalized strict pseudocontractions in Hilbert spaces. Fixed Point Theory and Applications 2014, 2014:66

\section{Submit your manuscript to a SpringerOpen ${ }^{\ominus}$ journal and benefit from:}

- Convenient online submission

- Rigorous peer review

- Immediate publication on acceptance

Open access: articles freely available online

- High visibility within the field

- Retaining the copyright to your article 